\title{
Vascular Steal Syndrome, Optic Neuropathy, and Foreign Body Granuloma Reaction to Onyx-18 Embolization for Congenital Orbito-Facial Vascular Malformation
}

\author{
Catherine Y. Liu Marc A. Yonkers Tiffany S. Liu Don S. Minckler \\ Jeremiah P. Tao \\ Gavin Herbert Eye Institute, University of California Irvine, Irvine, Calif., USA
}

\section{Key Words}

Vascular lesion · Embolization · Ptosis

\begin{abstract}
A 34-year-old patient presented with a right orbito-facial mass since childhood, consistent with a congenital arteriovenous (AV) malformation. Prior to presentation, she had multiple incomplete surgical resections and embolizations with N-butyl acetyl acrylate and Onyx-18. The patient reported gradual, progressive vision loss shortly after Onyx-18 embolization. Five months after embolization, she presented with decreased vision, disfigurement and mechanical ptosis relating to a large subcutaneous mass affecting the medial right upper eyelid and forehead. Significant exam findings included a visual acuity of 20/400 (20/60 prior to embolization), an afferent pupillary defect, and optic disc pallor. MRI and angiography revealed a persistent AV malformation with feeders from the ophthalmic artery and an absent choroidal flush to the right eye. Pathology from surgical resection showed a significant foreign body giant cell reaction to the embolization material adjacent to the vessels. We suggest that an incomplete embolization with Onyx-18 may have caused vascular steal syndrome from the ophthalmic artery.

(c) 2016 S. Karger AG, Basel
\end{abstract}

\section{Introduction}

Onyx-18 [1] is a nonadhesive liquid embolic agent increasingly being used for embolization of acquired orbital arteriovenous (AV) fistulas [1] and orbital tumors [2]. Congenital orbital AV malformations are rare and are composed of both open and hidden shunts [3]. Despite excellent results of Onyx-18 embolization in AV malformations of the brain [2], acquired AV fistulas [1], and orbital tumors [2], the results of Onyx-18 in treating congenital orbito-facial AV malformations are uncertain. Descriptions of ophthalmic complications associated with Onyx-18 are scarce in the literature. Inflammation and ischemia due to Onyx-18 may be problematic in the periocular region. Here, we present a case of vascular steal syndrome and subsequent optic neuropathy after Onyx18 embolization for a congenital palpebral and forehead AV malformation.

\section{Case Description}

A 34-year-old healthy female presented with a right supraorbital mass since childhood and gradual, progressive vision loss of the right eye starting 5 months prior to presentation. During childhood in India, she underwent resection of the mass (these records

\section{KARGER}

E-Mail karger@karger.com

www.karger.com/oop
(C) 2016 S. Karger AG, Basel

2296-4681/16/0023-0185\$39.50/0
Catherine Y. Liu, MD, PhD

Department of Ophthalmology, University of California Irvine 850 Health Sciences Road Irvine, CA 92697 (USA)

E-Mail yunxianl@uci.edu 

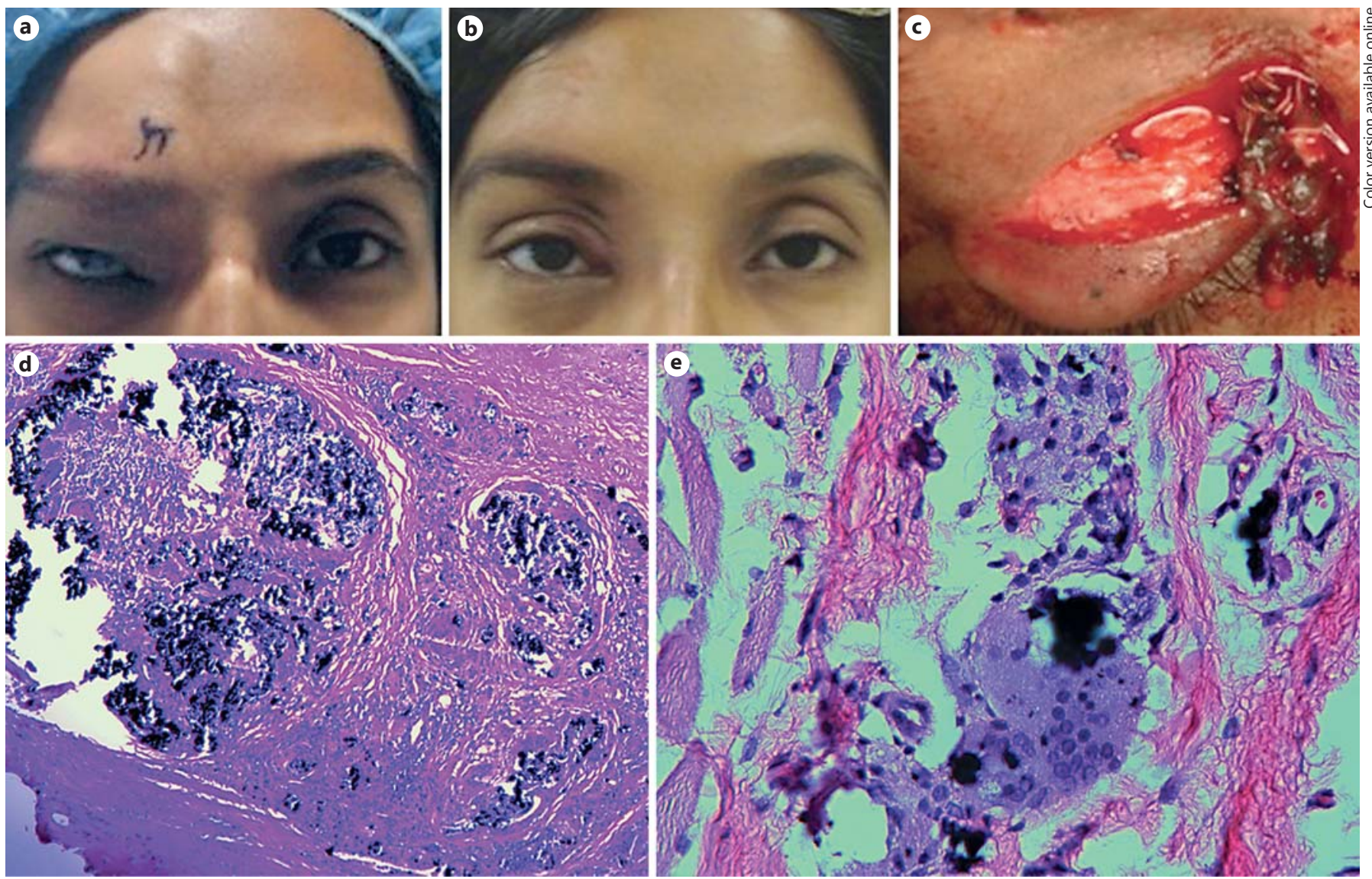

Fig. 1. External photographs and pathology of the right forehead, brow, and AV malformation of the eyelid. a Preoperative photograph. b Postoperative photograph. c Intraoperative photograph of the AV malformation of the right upper eyelid 5 months after Onyx-18 embolization via the ophthalmic artery and nasal vein. d Photomicrograph from orbital tissues: this biopsy demonstrates Onyx-18 particles impacted in and partially filling variably sized

were not available), but subsequently experienced a slow and continued growth of the mass. At the age of 29 years, workup was consistent with a vascular lesion, and she was treated elsewhere with embolization with N-butyl acetyl acrylate (NBCA) glue and resection. The lesion continued to grow over the next 5 years, and she underwent a second embolization with Onyx-18 and platinum microcoil. Both superselective arterial embolization and retrograde venous embolization were conducted by catheter access just distal to the ophthalmic artery and the nasal vein. A single platinum microcoil was placed in a draining vein.

Visual acuity 1 month prior to Onyx-18 embolization was $20 / 60$ in the right eye and stable. Visual acuity in the left eye was 20/20. Amblyopia accounted for the relatively decreased vision in the right eye. Following embolization, she experienced gradual vision decline, and presented to our institution 5 months after embolization for evaluation of the orbito-facial disfigurement due to the mass, right upper eyelid ptosis, and decreased vision. Her visual acuity at this time was 20/400 in the right eye. Flashlight test- small vessels, probably expanded venues, including numerous multinucleated giant cells in a fibrovascular matrix. Hematoxylin and eosin $(\mathrm{HE})$, original magnification $\times 100$. e Higher power photomicrograph demonstrating multinucleated giant cells that have phagocytosed Onyx-18 particles from the same specimen. HE, original magnification $\times 400$.

ing demonstrated a right relative afferent pupillary defect. External exam showed a soft, nontender, slightly purpuric superior nasal orbito-facial mass extending from just above the brow to the medial canthus on the right. Mechanical ptosis of the right upper lid was present (fig. 1a). Fundoscopy was significant for right optic nerve pallor.

An MRI with contrast revealed an enhancing vascular lesion of the right upper eyelid with many internal flow voids and early venous drainage, consistent with an AV malformation (fig. 2a). Arterial catheterization showed feeders from both the external and internal carotid systems, including the ophthalmic artery, and an area of incomplete embolization (fig. 2b, c). On catheterization, no choroidal flush was present in the right eye, but it was observed on the contralateral side. To correct the cosmetic disfigurement, the patient accepted a debulking procedure through a bicoronal incision combined with an eyelid crease incision. She was informed that her vision was not expected to improve after these reconstructive procedures. Intraoperative gross examina- 

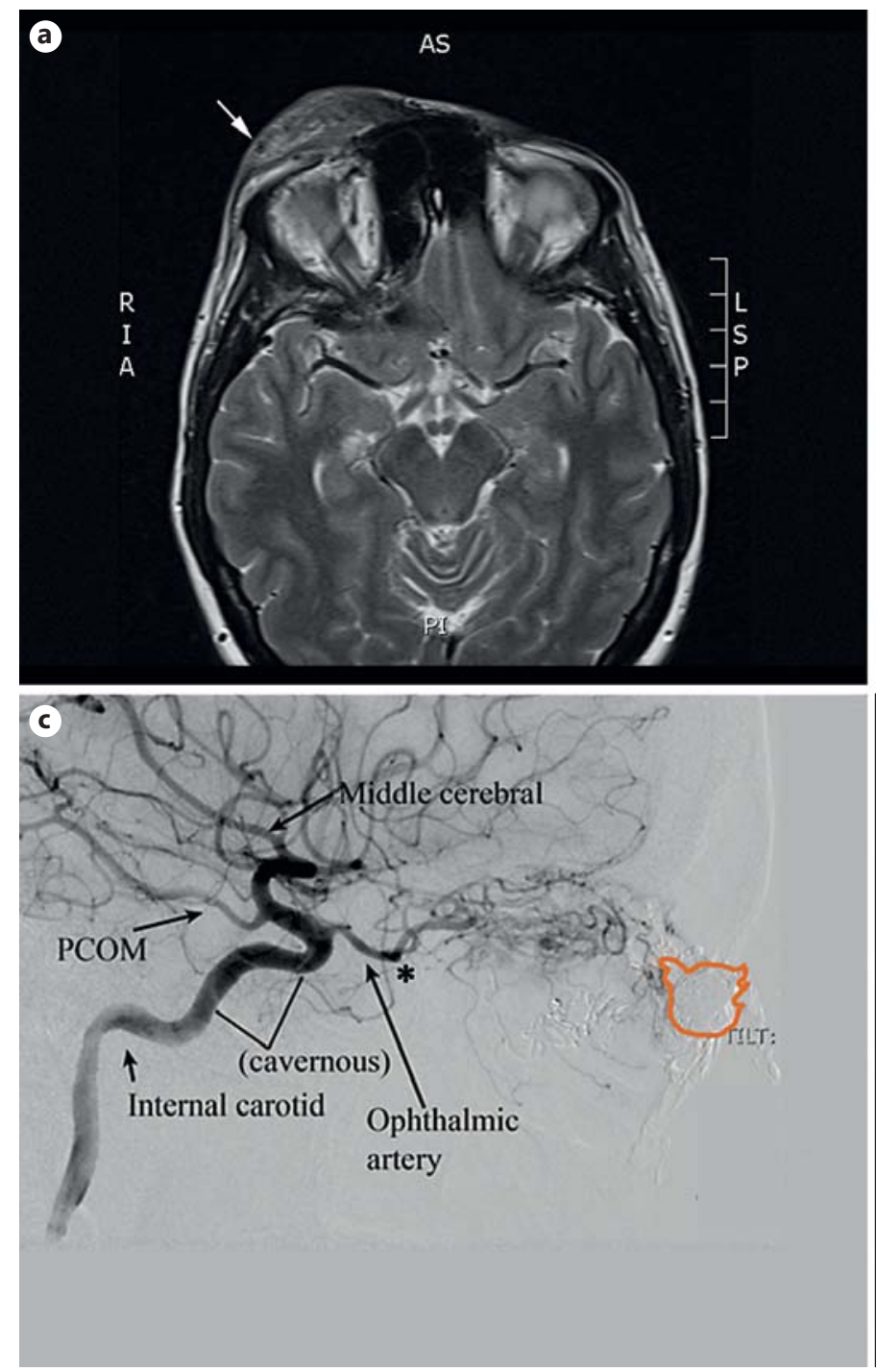

Fig. 2. Presentation of AV malformation. a MRI with gadolinium shows an enhancing mass of the right eyelid with many internal flow voids, one of which is indicated by an arrow. b The patient's external carotid system with feeders (asterisks) contributing to the $\mathrm{AV}$ malformation. The area of partial embolization is marked in

tion showed a vascular mass with a blue-green color, excised by sharp dissection (fig. 1c). Pathology demonstrated black pigmented material with significant foreign body giant cell reaction adjacent to myriad, variably sized vascular structures, consistent with an inflammatory reaction to retained embolization material (fig. 1d, e).

The patient subsequently underwent ptosis repair with levator advancement and further superior medial orbital soft-tissue debulking. She was very satisfied with the cosmetic results (fig. 1b). Her visual acuity remained stable and she had no signs of recurrence 1-year postsurgery.

Granuloma and Vascular Steal Syndrome due to Onyx-18 Embolization

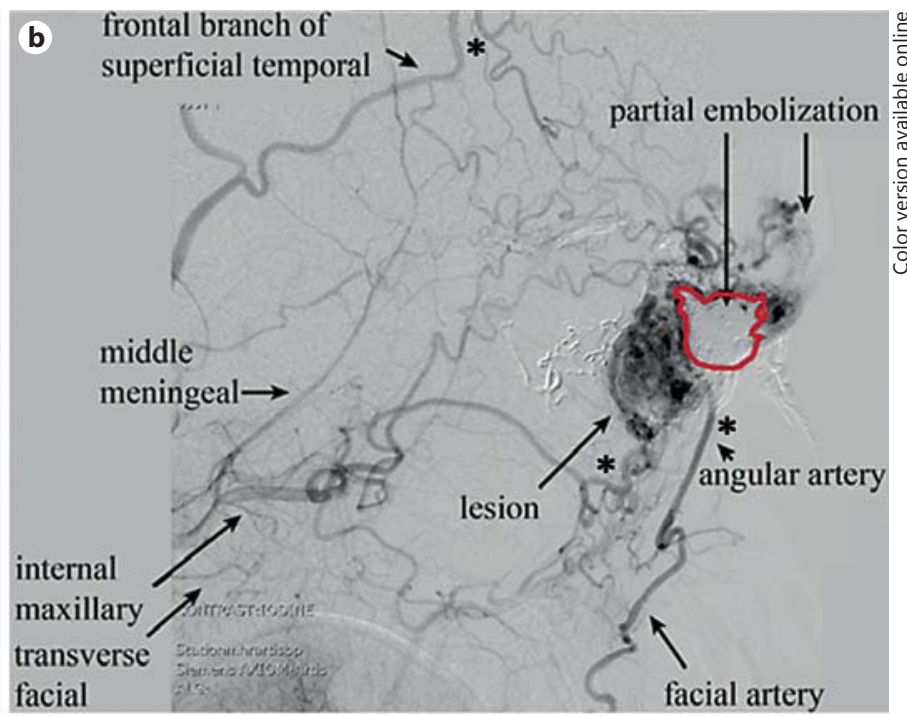

d

\section{Prior to embolization}
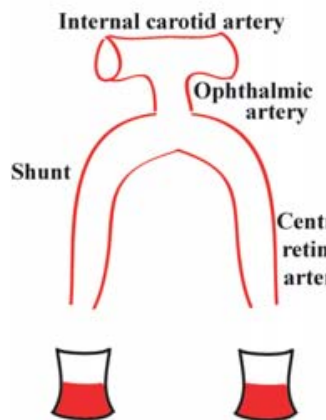

AV malformation

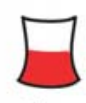

Eye

\section{Vascular steal after} incomplete embolization

Internal carotid artery

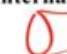

O artery

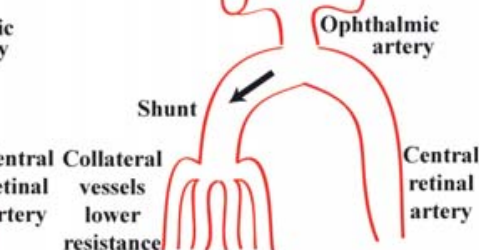
resistance $\mid)((1)$

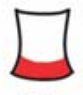

AV malformation Eye

red for orientation. c The patient's internal carotid system with feeders from the ophthalmic artery (asterisk). The area of partial embolization is highlighted in orange for orientation. d Schematic of the vascular steal syndrome mechanism in this patient. PCOM $=$ Posterior communicating artery.

\section{Discussion}

Anatomic classification governs the best management of orbito-facial vascular malformations [4]. This congenital vascular AV malformation differentiates from a noninvoluting congenital hemangioma owing to early venous drainage on MRI and relentless recurrence. Both vascular lesions are rare [5-7], but published reports show distinctly different responses to treatment. Whereas noninvoluting congenital hemangiomas can be removed in a 
stepwise approach with good outcomes, congenital AV malformations necessitate complete removal $[4,8]$. Incomplete excision or embolization can lead to patency of hidden channels and recurrence [9]. In our patient, incomplete embolization may have opened vascular feeders from the ophthalmic artery, creating a vascular steal syndrome (fig. 2d) [10].

Congenital AV malformations also differ from acquired AV fistulas. Acquired AV fistulas display a direct communication between arterial and venous systems, while congenital AV malformations consist of numerous AV shunts. AV fistulas can be treated directly by occlusion of feeder vessels with generally good outcomes [11]. In contrast, congenital AV malformations are more difficult to manage due to the numerous unseen vascular channels.

Though Onyx has been used to successfully treat meningiomas [2], carotid cavernous fistulas [12], and intraorbital AV fistulas [1], it is rarely used to treat congenital AV malformations of the eyelid. Owing to the inherent difference in anatomy and behavior of AV malformations, outcomes may not necessarily extrapolate. To our knowledge, only 1 case of palpebral AV malformations treated with Onyx-18 has been reported in the literature [6]. That case showed no complications with embolization of the distal segment of the ophthalmic artery and superficial temporal artery [6].

Interestingly, our patient experienced a decreased visual acuity after Onyx-18 embolization, but not after NBCA embolization. Several features differentiate these two agents. Onyx-18 is a nonadhesive liquid agent that solidifies quickly upon injection [13] and occludes vessels with a 5- $\mu$ m diameter. In contrast, NBCA occludes vessels with a $20-\mu \mathrm{m}$ diameter [14]. This allows Onyx-18 access to smaller caliber orbital vessels. Chronic foreign body giant cell reaction has been reported in over $50 \%$ of cases using Onyx-18, while none has been reported with NBCA [14]. Furthermore, Onyx-18 has a rate of recanalization of $18 \%$, while there is none after NBCA [14]. In our case, we speculate on the possibility that inflammation coupled with recanalization contributed partially to the opening of feeder channels from the ophthalmic artery. Of note, platinum microcoils can also cause an inflammatory reaction $[15,16]$, although only one was placed in our patient and is unlikely to be the major cause of the inflammatory reaction.

Alternative explanations for the gradual, progressive vision loss include a retrograde flow of embolic material into the ophthalmic artery, though fundoscopic and angiographic views were negative for emboli. Additionally, inadvertent occlusion of the ophthalmic artery by embolic agents would present more acutely with sudden vision loss [17]. Hemorrhagic events from necrosis of tissue have been reported [3], though this is unlikely due to the subacute onset, absence of pain, and absence of physical exam findings, consistent with this pathophysiology.

Limitations of this study include the observational nature of this single case report and dependence on the patient's recount of history due to the inability to obtain her complete set of medical records prior to her presentation to our institute. Additionally, ophthalmologic records available from prior to our involvement were exceedingly sparse. Preoperative angiography data would have been useful to review for locations of feeder vessels for comparison with our postoperative data. Although not performed in our patient, fluorescein angiography can show delayed choroidal filling [10].

In summary, early recognition of congenital AV malformations is important in management decisions. Embolization alone may not be sufficient to occlude all shunts. This case demonstrates the ophthalmological risks with use of these embolic agents in the orbito-facial zone. Further study is necessary to clarify the uses and risks of Onyx-18 embolization in congenital AV malformations.

\section{Acknowledgement}

This study was supported in part by an institutional Research to Prevent Blindness Grant.

\section{Statement of Ethics}

The subject of this study has given informed consent, and this work is compliant with our university's Institutional Review Board requirements.

\section{Disclosure Statement}

The authors have no financial or conflicts of interest to disclose. 


\section{References}

1 Lin CJ, Blanc R, Clarencon F, Piotin M, Spelle L, Williams M, Moret J: Transvenous embolization of an intraorbital arteriovenous fistula using Onyx. J Clin Neurosci 2010;17:783785.

2 Trivelatto F, Nakiri GS, Manisor M, Riva R, Al-Khawaldeh M, Kessler I, Mounayer C: Preoperative onyx embolization of meningiomas fed by the ophthalmic artery: a case series. AJNR Am J Neuroradiol 2011;32:1762-1766.

3 Warrier S, Prabhakaran VC, Valenzuela A, Sullivan TJ, Davis G, Selva D: Orbital arteriovenous malformations. Arch Ophthalmol 2008;126:1669-1675.

4 Rootman J, Heran MK, Graeb DA: Vascular malformations of the orbit: classification and the role of imaging in diagnosis and treatment strategies*. Ophthal Plast Reconstr Surg 2014; 30:91-104.

5 Krol A, MacArthur CJ: Congenital hemangiomas: rapidly involuting and noninvoluting congenital hemangiomas. Arch Facial Plast Surg 2005;7:307-311.

6 Clarencon F, Blanc R, Lin CJ, Mounayer C, Galatoire O, Morax S, Moret J, Piotin M: Combined endovascular and surgical approach for the treatment of palpebral arteriovenous malformations: experience of a single center. AJNR Am J Neuroradiol 2012;33:148153.
7 Kohout MP, Hansen M, Pribaz JJ, Mulliken JB: Arteriovenous malformations of the head and neck: natural history and management. Plast Reconstr Surg 1998;102:643-654.

8 Enjolras O, Mulliken JB, Boon LM, Wassef M, Kozakewich HP, Burrows PE: Noninvoluting congenital hemangioma: a rare cutaneous vascular anomaly. Plast Reconstr Surg 2001; 107:1647-1654

9 Trombly R, Sandberg DI, Wolfe SA, Ragheb J: High-flow orbital arteriovenous malformation in a child: current management and options. J Craniofac Surg 2006;17:779-782.

10 Andracchi S, Kupersmith MJ, Nelson PK, Slakter JS, Setton A, Berenstein A: Visual loss from arterial steal in patients with maxillofacial arteriovenous malformation. Ophthalmology 2000;107:730-736.

11 de Keizer R: Carotid-cavernous and orbital arteriovenous fistulas: ocular features, diagnostic and hemodynamic considerations in relation to visual impairment and morbidity. Orbit 2003;22:121-142.

12 Lv X, Li Y, Yang X, Jiang C, Wu Z: Endovascular management of direct carotid-cavernous sinus fistulas. Neuroradiol J 2012;25:130134.

13 Onyx LES. http://www.ev3.net/neuro/us/ liquid-embolics/onyx-liquid-embolicsystem.htm (accessed March 25, 2014).
14 Natarajan SK, Born D, Ghodke B, Britz GW, Sekhar LN: Histopathological changes in brain arteriovenous malformations after embolization using Onyx or N-butyl cyanoacrylate. Laboratory investigation. J Neurosurg 2009;111:105-113.

15 Byrne JV, Hope JK, Hubbard N, Morris JH: The nature of thrombosis induced by platinum and tungsten coils in saccular aneurysms. AJNR Am J Neuroradiol 1997;18:2933.

16 Killer M, Arthur AS, Barr JD, Richling B, Cruise GM: Histomorphology of thrombus organization, neointima formation, and foreign body response in retrieved human aneurysms treated with hydrocoil devices. J Biomed Mater Res B Appl Biomater 2010;94: 486-492.

17 Turner T, Trobe JD, Deveikis JP: Sequential branch retinal artery occlusions following embolization of an intracranial meningioma. Arch Ophthalmol 2002;120:857-860. 